\title{
Space-Time and Hadrons
}

\author{
C. C. Barros, Jr \\ Instituto de Física, Universidade de São Paulo, \\ C.P. 66318, 05315-970, São Paulo, SP, Brazil
}

Received on 29 September, 2006

\begin{abstract}
The hadronic structure is investigated in terms of a recently proposed theory which considers the effect of the strong interaction in the space-time description. From the equations, the masses of the particles may be calculated, and quark confinement appears as a natural result, without the need of confining potentials. Some properties of the considered particles, such as the topponium size, are also estimated.
\end{abstract}

Keywords: Quark confinement; Heavy quarkonium; Space-time curvature

The effect of space-time in quantum physics, or, semantics apart, the effect of quantum physics in the structure of spacetime are subjects that until today do not have a theory. The efforts made in the attempt of quantizing the gravitational field illustrate this fact.

In most of the descriptions of quantum systems and of particle physics, for example, this question is not considered, and the space is the flat Minkowski one, with the metric tensor

$$
g_{\mu \nu}=(1,-1,-1,-1),
$$

and no interaction is considered to affect its structure. However a fundamental question that remains is whether the electromagnetic and strong interactions may affect space-time, in a way similar to the role of the gravitational field in general relativity, and if these effects may be observed.

With these questions in mind, recently, a theory has been proposed [1]-[3], in which the effects mentioned above have been taken into account, and very interesting results have been obtained. For example, the agreement with the experimental results of the deuterium atom spectrum is improved by one decimal digit, when compared with the Dirac results [4]-[6], a fact that may hardly be considered as a coincidence, and this agreement shows the same pattern for all energy levels. The results of the theory when applied to hadron physics are also very interesting, and quark confinement appears in a natural way.

In this work a brief review of this theory will be made, and a special emphasis will be given to its application to the study of hadrons. The fundamental principle of the theory will also be formulated.

For simplicity, a first step is to consider a system with spherical symmetry, but the basic ideas can be generalized to systems with arbitrary metrics. In fact, this approach is very illustrative, many systems may be described by spherical symmetry, or at least, investigated in a first approximation. With this procedure, the main results of the theory are obtained and all the results may be obtained analytically.

So, if the spherical symmetry is considered, the space-time is described by the metric derived in [1], that is very similar to the Schwarzschild metric [7],[8],

$$
d s^{2}=\xi d \tau^{2}-r^{2}\left(d \theta^{2}+\sin ^{2} \theta d \phi^{2}\right)-\xi^{-1} d r^{2},
$$

where $r, \theta$ and $\phi$ are the particle coordinates, $\xi(r)$ is determined by the interaction potential $V(r)$ and is a function only of $r$, for a time independent interaction.

The energy relation for this system is [1]

$$
\frac{E}{\sqrt{\xi}}=\sqrt{p^{2} c^{2}+m_{0}^{2} c^{4}},
$$

with

$$
\xi^{\frac{1}{2}}=1+\frac{V}{m c^{2}}
$$

Applying these ideas to the study of one electron atoms, $V$ is the Coulomb potential

$$
V(r)=-\frac{\alpha Z}{r},
$$

$\alpha$ is the fine structure constant and $Z$ is the atomic number. Consequently, the function $\xi$ is given by

$$
\xi=1-\frac{2 \alpha Z}{m c^{2} r}+\frac{\alpha^{2} Z^{2}}{m^{2} c^{4} r^{2}},
$$

where $m$ is the electron mass. These expressions determine the horizon of events at $r_{0}$, that appears from the metric singularity $\xi\left(r_{0}\right)=0$, and using the values of [9], one finds

$$
r_{0}=\frac{\alpha Z}{m c^{2}}=2.818 Z \mathrm{fm},
$$

that is not a negligible value at the atomic scale.

In a space-time described by the metric (2), the quantum wave equation for spin-1/2 particles is [1]

$$
\frac{i \hbar}{\xi} \frac{\partial}{\partial t} \Psi=\left(-i \hbar c \vec{\alpha} \cdot \vec{\nabla}+\beta m_{0} c^{2}\right) \Psi
$$

where $\Psi$ is a four-component spinor.

The spacial part of $\Psi$, that is a four component spinor, may be written as

$$
\psi=\left(\begin{array}{c}
F(r) \chi_{\kappa}^{\mu} \\
i G(r) \chi_{-\kappa}^{\mu}
\end{array}\right)
$$

with the aid of the usual two component spinors, $\chi_{\kappa}^{\mu}$ [1], where

$$
\begin{aligned}
& \kappa=l \quad \text { for } j=l-1 / 2, \\
& \kappa=-l-1 \text { for } j=l+1 / 2 .
\end{aligned}
$$


The radial part of eq. (8) may be rewritten as a pair of coupled equations for the and the $F$ and $G$ functions

$$
\begin{aligned}
& \sqrt{\xi} \frac{d F}{d r}+(1+k) \frac{F}{r}=\left(\frac{E}{\sqrt{\xi}}+m_{0}\right) G \\
& \sqrt{\xi} \frac{d G}{d r}+(1-k) \frac{G}{r}=-\left(\frac{E}{\sqrt{\xi}}-m_{0}\right) F .
\end{aligned}
$$

Considering a Coulomb-like potential, the $\xi$ function becomes

$$
\xi=\left(1-\frac{\alpha Z}{m_{0} c^{2} r}\right)^{2},
$$

and inserting it (12) in eq. (11) and making the substitution $\rho=\beta r$, the equations may be put in the form

$$
\begin{aligned}
& \xi \frac{d F}{d \rho}+\sqrt{\xi}(1+k) \frac{F}{\rho}=\left(\frac{E}{\beta}+\sqrt{\xi} \frac{m_{0}}{\beta}\right) G \\
& \xi \frac{d G}{d \rho}+\sqrt{\xi}(1-k) \frac{G}{\rho}=-\left(\frac{E}{\beta}-\sqrt{\xi} \frac{m_{0}}{\beta}\right) F,
\end{aligned}
$$

for $r \neq r_{0}$. Solving these equations, what may be done using the Frobenius method, the relation for the energy levels is determined

$$
E_{N}= \pm m_{e} c^{2} \sqrt{\frac{1}{2}-\frac{N^{2}}{8 \alpha^{2}} \pm \frac{N}{4 \alpha} \sqrt{\frac{N^{2}}{4 \alpha^{2}}+2}}
$$

where the physical values are the positive ones.

At a first sight equation (8) may seem very similar to the Dirac equation, but it shows some important differences. The first one is the numerical aspect. Applying (14) to the deuterium atom [1], the agreement with the experimental values of the energy levels is improved in comparison with the Dirac spectrum and shows deviations from the data of the order of $0.005 \%$. Another characteristic of the general quantum mechanics is the existence of the horizon of events at $r_{0}$. In the hydrogen atom, this fact is not important, $r_{0} \sim 2.8 \mathrm{fm}$, and, from the solution of (8), an electron with energy of the order of few $\mathrm{eV}$ has a very small probability of being found in this region, so, in practical terms, no effect is observed. Although, for heavy elements $r_{0}$ increases and this effect is not negligible [3].

Now let us turn our attention to the study of hadrons, that today, we believe that are systems composed of quarks, which interact via gluon fields and many authors suppose that the mean effect of these interactions may be represented by selfconsistent effective potentials. In the Bogolioubov [10] and in the MIT bag model [11] the constituent quarks are Dirac particles, confined by a spherical step potential (with $V_{0} \rightarrow \infty$ ). Other models consider different shapes for the potential, as for example $r^{n}$ terms [12]- [14] and the Cornell model [15], [16], that uses a linear plus strong Coulomb potential

$$
V=\frac{a}{r}+b r
$$

\begin{tabular}{|c|c|c|c|c|c|}
\hline & $m$ & $\alpha$ & $r_{0}(\mathrm{fm})$ & $M$ & $M_{\text {exp }}$ \\
\hline & 0.38 & 1.60 & 0.83 & 0.938 & 0.938 \\
\hline$J / \psi(c \bar{c})$ & 1.79 & 1.00 & 0.11 & 3.10 & 3.10 \\
\hline$\Upsilon(b \bar{b})$ & 5.50 & 1.05 & 0.05 & 9.47 & 9.46 \\
\hline$t \bar{t}$ & 174 & 1.00 & 0.002 & 310 & - \\
\hline
\end{tabular}

TABLE I: Values of the masses $M(\mathrm{GeV})$ of the hadrons, composed of constituent quarks of mass $m(\mathrm{GeV})$ compared with the experimental ones [9]. The calculations are made with eq. (14), obtained for Coulomb potentials with coupling $\alpha$.

We may construct a model, supposing that the quarks inside the hadrons are described by eq. (8), and the mean effect of the interaction is given by a strong Coulomb potential (a Coulomb potential with $\alpha \sim 1$ ). In a baryon, for example, the mass is given by

$$
M=\sum_{i=1}^{3} E_{N}\left(q_{i}\right)
$$

with $E_{N}$ from eq. (14). Some numerical results may be found in Table 1. Observing the Table, one must note that $t \bar{t}$ and $b \bar{b}$ must be very small mesons. In the topponium, for example, $r_{t \bar{t}} \sim 0.002 \mathrm{fm}$. One must also note that the agreement with experimental data is very good.

The wave functions of the $i$ quarks are determined by eq. (8). An important result of this approach is that quark confinement occurs without the need of a confining potential, as the wave functions of the quarks at $r_{0}$ are $\Psi\left(r_{0}\right)=0$. This discontinuity at the horizon of events determines a strong confinement.

The horizon of events is not an exclusive feature of the Coulomb potential, it may appear for any attractive potential, when the condition

$$
\xi\left(r_{0}\right)=0,
$$

is satisfied, what occurs for

$$
V\left(r_{0}\right)=-m c^{2}
$$

One must remark that these preliminary estimates, just considering a strong Coulomb potential, provided very accurate results, and even the magnetic moments calculated this way are very accurate [1]. However we think that a better shape of the potential must be considered in order to describe all the baryons and mesons, and this is what is being done. A reliable description of the strong interactions must be based on a short-ranged potential as, for example, a Woods-Saxon one and we must note that changing the shape of the potential, the main results presented here will not change.

In this work, we showed how to introduce the effect of the strong and electromagnetic interactions in the metric of space time and that this effect is important in order to understand the quantum world and specially the hadronic structure.

The mathematical formulation presented in this work, is the result of the following principle: different particles see the space-time in different ways, or alternatively, different particles are affected by the space-time in different ways. So, what 
is being said, is that with this approach, an absolute space, to every particles, as it appears in the general relativity, does not exist. When we deal with quantum systems, the differences appear. For example, a quark sees a space curved by the strong gluonic field of another quark and, in fact, this quark is confined by a trapping surface, as defined by Penrose [17]. However this space is flat for a photon or for a lepton. There is no absurd in this fact and it is observable, for example, in a radiative decay of a hadron,

$$
H \rightarrow H^{\prime} \gamma
$$

The process may be understood in terms of an excited quark $q^{*}$ that decays

$$
q^{*} \rightarrow q \gamma
$$

producing a photon $\gamma$, the quark remains confined as the photon is emitted and escapes from the strong horizon of events. This also supports the fact that it is a good idea to use leptons in order to probe the interior of the hadrons as they are not affected by the strong horizon of events.

It must be remarked that the theory presented in this work is is still in an initial stage of development. Many aspects, even in the foundations of the theory must be further examined. Systems that do not present spherical symmetries, although important in many physical situations have not been studied yet, and different metrics must be considered. Other kinds of interactions, such as a vector potential have not been included, for simplicity, but it may be done by introducing then in the energy-momentum tensor. Many of these questions will be addressed in future works.

Despite of these limitations, in all of the studied systems, the results obtained are very good and totally consistent with experiment. This motivates the continuation of the studies in this field.

Another important point that must be emphasized is that the theory presented is a quantum one, that is, a theory for very small objects. Any incautious reader should think that it contradicts general relativity. This is right in the same sense that quantum theory contradicts Newtonian mechanics. Taking the classical limit of the presented theory for large (macroscopic) masses, general relativity is recovered and the effects presented in this paper vanish. From the point of view of this work, general covariance does not occur in quantum systems, it must be considered to be a classical property.
[1] C. C. Barros Jr., Eur. Phys. J. C 42, 119 (2005).

[2] C. C. Barros Jr., Eur. Phys. J. C 45, 421 (2006).

[3] C. C. Barros Jr., physics/0609115 (2006).

[4] P. A. M. Dirac, Proc. Roy. Soc. Lon. A 117, 610 (1930); P. A. M. Dirac, ibid A 126, 360 (1930).

[5] L. I. Schiff, Quantum mechanics, (McGraw-Hill, Singapore, 1968).

[6] A. Sommerfeld, Ann. Physik. 51, 1 (1916).

[7] L. Landau and E. Lifshitz, The Classical Theory of Fields, (Pergamon, Oxford, 1975).

[8] S. Weinberg, Gravitation and cosmology: principles and applications of the general theory of relativity, (John Wiley and Sons, New York, 1972).

[9] Particle Data Group, S. Eidelman et al., Phys. Lett. B 592, 1 (2004).
[10] P. N. Bogolioubov, Ann. Inst. Henri Poincaré, 8, 163 (1967).

[11] A. Chodos, R. L. Jaffe, K. Johnson, C. B. Thorn, and V. Weisskopf, Phys. Rev. D 9, 3741 (1974); A. Chodos, R. L. Jaffe, K. Johnson, and C. B. Thorn, Phys. Rev. D 10, 2599 (1974).

[12] R. Tegen, Ann. Phys. 197, 439 (1990).

[13] P. L. Ferreira, J. A. Helayel, and N. Zagury, Nuovo Cim. A 55, 215 (1980)

[14] N. Isgur and G. Karl, Phys. Rev. D 18, 4187 (1978); D 19, 2653 (1979).

[15] E. Eichten, K. Gottfried, T. Kinoshita, K. D. Lane, and T. -M. Yan, Phys. Rev. 17, 3090 (1978); 21, 203 (1980).

[16] E. Eichten and F. Feinberg, Phys. Rev. D 23, 2724 (1981).

[17] R. Penrose, Phys. Rev. Lett. 14, 57 (1965); Riv. Nuovo Cim. Speciale, 252 (1969). 